\title{
A glosa odontológica em uma operadora de grupo de grande porte
} Dental services disallowance in a major health plan provider

\author{
Geraldo Elias Miranda \\ Marta Cristina Portes Siqueira** \\ Ricardo Luiz Siqueira Magalhães Ferreira** \\ Fernanda Capurucho Horta Bouchardet ${ }^{* * *}$ \\ Duarte Nuno Pessoa Vieira*** \\ Eduardo Daruge Júnior ${ }^{* * * * *}$
}

\section{Resumo}

Objetivo: verificar a frequência de glosa total e dos procedimentos odontológicos realizados pela rede credenciada de uma operadora de plano de saúde. Materiais e método: trata-se de um trabalho observacional transversal no qual foi realizado um levantamento retrospectivo em uma operadora de grupo exclusivamente odontológica no terceiro trimestre de 2012. A análise quantitativa agrupou os procedimentos baseando-se no rol da Agência Nacional de Saúde Suplementar. Os dados coletados foram tabulados e submetidos a análise estatística com intervalos com 95\% de confiança e nível de significância de 5\%. Resultados: dos 264.758 procedimentos faturados, 21.716 foram glosados; portanto, o percentual global de glosas em relação ao total de procedimentos foi de $8,20 \%$. A maior frequência de glosas foi observada na radiologia (35,93\%), seguida pela dentística $(24,04 \%)$ e pela prevenção $(16,70 \%)$. Do total de glosas, a endodontia e a prótese tiveram $4,77 \%$ e $2,32 \%$, respectivamente. Entretanto, quando se analisou cada grupo do rol individualmente, $24,37 \%$ dos procedimentos da prótese e $22,23 \%$ dos procedimentos da endodontia foram glosados. Conclusão: a frequência total de glosa na operadora de plano de saúde foi $8,20 \%$. Os procedimentos mais glosados foram de radiologia, dentística e prevenção. Quando se avaliou a frequência de glosa dentro de um mesmo grupo, a prótese e a endodontia foram as especialidades mais glosadas.

Palavras-chave: Auditoria odontológica. Saúde suplementar. Avaliação dos serviços de saúde. Odontologia Legal.

\section{Introdução}

O setor de saúde suplementar, regido pela Lei 9.656/98 e fiscalizado pela Agência Nacional de Saúde Suplementar (ANS), expandiu sua atuação no campo da saúde e tornou-se imprescindível para a assistência da população brasileira. O Brasil possui o segundo maior sistema privado de saúde do mundo ${ }^{1}$, havendo uma tendência de crescimento da odontologia no setor suplementar ${ }^{2}$.

O Estado fiscaliza e monitora a solvência das operadoras de planos de saúde (OPS). Manter o setor estável é uma grande preocupação do Governo, pois atualmente as OPS arcam com a assistência em saúde de uma parte significativa da população. A regulação da saúde suplementar ocorre em dois espaços distintos: no campo da macrorregulação e no da microrregulação. A macrorregulação é constituída pela legislação e regulamentação da ANS. A microrregulação ocorre na auditoria por meio dos protocolos estipulados pelas operadoras para os prestadores de serviços ${ }^{3}$.

A auditoria em saúde tem papel primordial de garantir a qualidade da assistência prestada e o respeito às normas técnicas, éticas e administrativas, previamente estabelecidas nos contratos entre prestadores de serviços e operadoras. A auditoria deve, também, manter a relação custo-benefício da assistência compatível e equacionada aos recursos

http://dx.doi.org/10.5335/rfo.v18i2.3406

Auditor odontológico, mestrando em Odontologia Legal FOP/UNICAMP, Piracicaba, SP, Brasil.

Auditor odontológico, especialista em Odontologia Legal, Belo Horizonte, MG, Brasil.

Coordenadora do curso de Odontologia Legal da PUC/MINAS, Belo Horizonte, MG, Brasil.

Professor doutor catedrático da Universidade de Coimbra, Coimbra, Portugal.

Livre-docente em Odontologia, professor titular de Odontologia Legal da FOP/UNICAMP, Piracicaba, SP, Brasil. 
financeiros disponíveis, contribuindo, assim, para a redução da taxa de sinistralidade das operadoras. A taxa de sinistralidade é calculada na relação entre as despesas assistenciais (médicas e odontológicas) e a contraprestação ${ }^{2}$.

O resultado de uma auditoria pode ser conforme quando o serviço está em concordância com as especificações administrativas (preenchimento e envio de documentação, elegibilidade e carência do beneficiário) e com o padrão de qualidade técnica. O serviço não conforme é aquele que está em desacordo com as especificações administrativas ou é tecnicamente inaceitável ${ }^{4}$.

A glosa é o cancelamento ou recusa parcial ou total de uma fatura apresentada pelo prestador de serviços à OPS diante de uma não conformidade do serviço prestado. A pode ser administrativa ou técnica. A glosa administrativa não está relacionada ao tratamento em si, mas sim à forma como a cobrança dos procedimentos é apresentada. As glosas técnicas estão relacionadas ao tratamento realizado, que pode ser considerado desejável, aceitável ou inaceitável ${ }^{5}$.

Com a expansão do setor de saúde suplementar, tornam-se necessárias pesquisas sobre os critérios de auditagem para a melhoria da assistência odontológica e a relação entre prestadores e operadoras. Além disso, segundo alguns autores ${ }^{6-7}$, há uma carência de estudos sobre o tema.

O objetivo deste trabalho foi verificar a frequência de glosa total e dos procedimentos odontológicos realizados pela rede credenciada de uma operadora de grupo de grande porte.

\section{Materiais e método}

Trata-se de um trabalho observacional transversal no qual foi realizado um levantamento retrospectivo em relatórios de faturamentos e glosas em uma operadora exclusivamente odontológica, modalidade odontologia de grupo de grande porte (acima de 100 mil beneficiários).

A amostra foi composta pelos credenciados que enviaram produção para auditagem no terceiro trimestre de 2012. Foi escolhido esse período por coincidir com os dados enviados pela operadora no arquivo Sistema de Informações de Produtos (SIP) à ANS. O SIP tem como finalidade acompanhar a assistência dos serviços prestados aos beneficiários de planos de saúde.

A análise quantitativa agrupou os procedimentos baseando-se no Rol de Procedimentos e Eventos em Saúde da ANS, que é dividido em: Diagnóstico, Urgência, Radiologia, Prevenção, Dentística, Periodontia, Cirurgia, Endodontia e Prótese.
O processo de auditagem procede à seguinte logística: a rede credenciada envia à operadora os atendimentos dos beneficiários registrados nas Guias de Tratamento Odontológico (GTO) e nas Guias de Serviço Profissional/Serviço Auxiliar de Diagnóstico e Terapia (SP/SADT), de acordo com o padrão utilizado na Troca de Informação em Saúde Suplementar (TISS).

Ao receber as guias para a cobrança, a OPS abre, no sistema de informação, um lote por prestador, em que são discriminados todos os procedimentos executados. O lote que representa o valor faturado à operadora é encaminhado para a auditoria verificar a conformidade dos procedimentos. A análise da conformidade abrange critérios técnicos e administrativos. $\mathrm{O}$ procedimento não conforme é glosado. Assim, os procedimentos faturados são a soma dos procedimentos pagos e dos glosados.

Caso o prestador não concorde com a glosa, poderá recorrer por meio de processo denominado "revisão de glosa", no qual nova apreciação do procedimento será realizada. Esta pesquisa não avalia os lotes de revisão de glosa, restringindo-se aos lotes de faturamento.

Esta pesquisa foi aprovada pelo Comitê de Ética em Pesquisa da FOP/UNICAMP sob o protocolo no $20 / 2013$ e não contém elementos que permitam a identificação dos prestadores ou beneficiários dos serviços.

Os dados coletados foram tabulados e submetidos a análises uni- e bi-variadas com o auxílio dos softwares SPSS 13.0 (Statistical Package for the Social Science ${ }^{\circledR}$, IBM, Chicago, USA) e Minitab 15 (Minitab Inc. ${ }^{\circledR}$, State College, USA).

Para caracterizar os resultados, utilizaram-se tabelas de contingência, apresentando as frequências e taxas de ocorrência das características estudadas, de forma a descrevê-las. Foram construídos intervalos com $95 \%$ de confiança, de maneira a levar em consideração o erro amostral nas estimativas obtidas. Para determinar se as diferenças e associações encontradas são estatisticamente significativas, utilizou-se o nível de significância de 5\%. 


\section{Resultados}

Os resultados do presente estudo são descritos nas Tabelas 1 a 3 e na Figura 1.

Tabela 1 - Frequências (absoluta e relativa) e intervalo de confiança dos procedimentos faturados

\begin{tabular}{|c|c|c|c|c|}
\hline \multirow{2}{*}{ Procedimento } & \multirow{2}{*}{$\begin{array}{c}\text { Frequência } \\
n\end{array}$} & \multirow{2}{*}{$\begin{array}{c}\text { Percentual } \\
\%\end{array}$} & \multicolumn{2}{|c|}{ IC 95\% } \\
\hline & & & Inf & Sup \\
\hline Radiologia & 110152 & 41,60 & 41,42 & 41,79 \\
\hline Prevenção & 64178 & 24,24 & 24,08 & 24,40 \\
\hline Dentística & 30587 & 11,55 & 11,43 & 11,68 \\
\hline Diagnóstico & 24442 & 9,23 & 9,12 & 9,34 \\
\hline Periodontia & 18773 & 7,09 & 6,99 & 7,19 \\
\hline Cirurgia & 7117 & 2,69 & 2,63 & 2,75 \\
\hline Endodontia & 4660 & 1,76 & 1,71 & 1,81 \\
\hline Urgência & 2781 & 1,05 & 1,01 & 1,09 \\
\hline Prótese & 2068 & 0,78 & 0,75 & 0,82 \\
\hline Total & 264758 & 100,00 & - & - \\
\hline
\end{tabular}

Tabela 2 - Frequências (absoluta e relativa) e intervalo de confiança dos procedimentos glosados

\begin{tabular}{|c|c|c|c|c|}
\hline \multirow{2}{*}{ Procedimento } & \multirow{2}{*}{$\begin{array}{c}\text { Frequência } \\
n\end{array}$} & \multirow{2}{*}{$\begin{array}{c}\text { Percentual } \\
\%\end{array}$} & \multicolumn{2}{|c|}{ IC 95\% } \\
\hline & & & $\operatorname{lnf}$ & Sup \\
\hline Radiologia & 7802 & 35,93 & 35,29 & 36,57 \\
\hline Dentística & 5220 & 24,04 & 23,47 & 24,61 \\
\hline Prevenção & 3627 & 16,70 & 16,21 & 17,20 \\
\hline Diagnóstico & 1386 & 6,38 & 6,06 & 6,72 \\
\hline Endodontia & 1036 & 4,77 & 4,49 & 5,06 \\
\hline Periodontia & 926 & 4,26 & 4,00 & 4,54 \\
\hline Cirurgia & 881 & 4,06 & 3,80 & 4,33 \\
\hline Prótese & 504 & 2,32 & 2,12 & 2,53 \\
\hline Urgência & 334 & 1,54 & 1,38 & 1,71 \\
\hline Total & 21716 & 100,00 & - & - \\
\hline
\end{tabular}

Tabela 3 - Frequências relativa e absoluta de glosas em relação aos procedimentos faturados

\begin{tabular}{|c|c|c|c|c|c|}
\hline \multirow{2}{*}{ Procedimento } & \multirow{2}{*}{$\begin{array}{c}\text { Glosas } \\
\mathrm{n}\end{array}$} & \multirow{2}{*}{$\begin{array}{l}\text { Procedimentos } \\
\mathrm{N}\end{array}$} & \multirow{2}{*}{$\begin{array}{c}\text { Percentual } \\
\%\end{array}$} & \multicolumn{2}{|c|}{ IC 95\% } \\
\hline & & & & Inf & Sup \\
\hline Prótese & 504 & 2068 & 24,37 & 22,53 & 26,28 \\
\hline Endodontia & 1036 & 4660 & 22,23 & 21,04 & 23,45 \\
\hline Dentística & 5220 & 30587 & 17,07 & 16,65 & 17,49 \\
\hline Urgência & 334 & 2781 & 12,01 & 10,82 & 13,28 \\
\hline Cirurgia & 881 & 7117 & 12,38 & 11,62 & 13,17 \\
\hline Radiologia & 7802 & 110152 & 7,08 & 6,93 & 7,24 \\
\hline Prevenção & 3627 & 64178 & 5,65 & 5,47 & 5,83 \\
\hline Diagnóstico & 1386 & 24442 & 5,67 & 5,38 & 5,67 \\
\hline Periodontia & 926 & 18773 & 4,93 & 4,63 & 5,25 \\
\hline Total & 21716 & 264758 & 8,20 & 8,10 & 8,31 \\
\hline
\end{tabular}




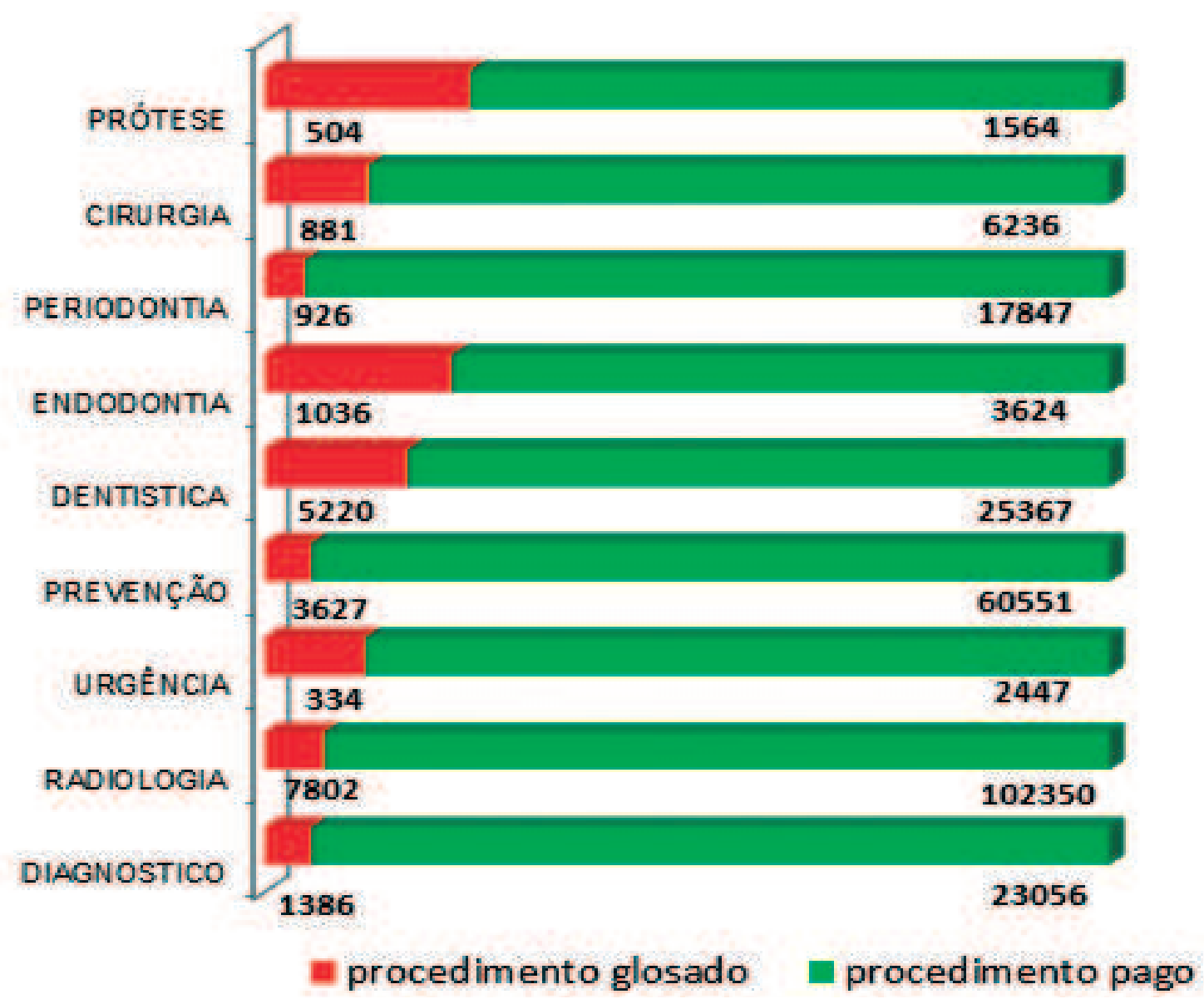

Figura 1 - Frequência absoluta de procedimentos glosados e de procedimentos pagos

\section{Discussão}

O percentual global de glosa em relação ao total de procedimentos foi de $8,20 \%$, variando de 8,10 a $8,31 \%$ com $95 \%$ de confiança, mostrando que, em geral, cerca de $8 \%$ dos procedimentos são glosados.

Uma pesquisa mostrou que a frequência de glosa em uma cooperativa odontológica foi de $14 \%^{6}$. Outro trabalho realizado no sistema DATASUS observou um aumento da glosa de menos de $5 \%$ no período de 2001 a 2007 para $14,1 \%$ em $2010^{7}$. Portanto, a quantidade de glosa no Sistema Único de Saúde e na cooperativa das pesquisas citadas foi maior do que a encontrada no presente trabalho.

Deve-se considerar que a frequência de glosa encontrada $(8,20 \%)$ poderá ser menor, pois não foram avaliadas as revisões de glosa. As revisões de glosa são aquelas situações em que o prestador corrige as imperfeições ou justifica uma conduta com base em parâmetros de consenso e submete a conta para nova apreciação. A revisão é considerada uma nova auditora e é avaliada por auditor diferente daquele que já tinha auditado o procedimento. Logo, o pagamento ao prestador referente à sua produção mensal poderá ser modificado caso a glosa não seja mantida.
As glosas não foram analisadas quantitativamente quanto ao tipo: administrativa ou técnica. As glosas administrativas podem ocorrer em qualquer procedimento e estão relacionadas aos critérios de elegibilidade do beneficiário para receber o atendimento, envio de documentação de atendimento fora do prazo, preenchimento incompleto ou rasurado das GTO e SP/SADT, dentre outros.

Do total de procedimentos faturados, os radiológicos representaram $41,60 \%$, os preventivos, $24,24 \%$ e os de dentística, $11,55 \%$, sendo esses dados significativamente diferentes, tendo em vista que os intervalos de confiança não apresentam interseção entre si, nem com os demais (Tabela 1).

A maior frequência de glosas foi observada na radiologia, com $35,93 \%$ dos casos, seguida pela dentística, com $24,04 \%$, e por procedimentos de prevenção, com 16,70\%, sendo esses dados significativamente diferentes dos demais (Tabela 2). A somatória desses grupos representa $77,03 \%$ do total de glosas, demonstrando que a quantidade de glosas é diretamente proporcional à quantidade de procedimentos faturados.

Segundo pesquisa, no SUS, a radiologia representa $44 \%$ dos procedimentos odontológicos executados, seguidos de $21,5 \%$ de procedimentos perio- 
dontais, $14,2 \%$ de endodônticos e $6,4 \%$ de restauradores $^{8}$. O resultado encontrado para procedimentos radiológicos é semelhante ao do presente trabalho. O segundo lugar em execução foi ocupado pelos procedimentos periodontais. A pesquisa realizada no SUS não considerou como categoria os procedimentos preventivos. Existe a possibilidade de que os procedimentos de profilaxia e raspagens supragengivais tenham sido alocados e contabilizados juntamente com os procedimentos periodontais.

Os procedimentos radiológicos foram os mais executados, pois uma radiografia corresponde a um procedimento. Os exames radiológicos executados ultrapassaram as solicitações da OPS, pois vários dentistas solicitam na consulta inicial série radiográfica completa (14 radiografias), bite-wing e panorâmica. As glosas técnicas que ocorrem nos exames complementares são devidas a imagens não visualizáveis: erro de técnica ou erro de processamento. Um trabalho realizado na Suécia mostrou que a qualidade radiográfica em $28 \%$ dos casos analisados foi considerada inaceitável para avaliação do tratamento proposto 9 .

Os procedimentos de dentística representaram $24,24 \%$ do total de glosas. Com relação aos procedimentos restauradores, o principal motivo da glosa observado estava relacionado com o diagnóstico de cárie. O prestador incluía em seu plano de tratamento a execução de restaurações em lesão incipiente de esmalte, sulco acastanhado e dente hígido. Esse tipo de sobretratamento verificado na prática odontológica de alguns cirurgiões-dentistas credenciados não segue o que preconiza a odontologia baseada em evidência científica.

Uma pesquisa realizada em uma OPS em que foram avaliados os planos de tratamento propostos pelos credenciados mostrou que 8,7 dentes seriam restaurados em cada usuário. Após auditoria, chegou-se a 4,8 dentes/usuário, ou seja, apenas $55,89 \%$ das restaurações propostas foram consideradas necessárias ${ }^{4}$.

Um estudo de coorte mostrou que há uma disparidade no plano de tratamento odontológico realizado por cirurgiões-dentistas que são assalariados e aqueles que recebem por produção. Em situações em que o cirurgião-dentista recebe por produção, o plano de tratamento propõe maior quantidade de procedimentos ${ }^{10}$.

Também foi verificada repetição do procedimento executado dentro do período de longevidade, indicando o insucesso do tratamento em dentística. Aprovar procedimentos com falhas técnicas acarreta custos para a OPS, credenciado e beneficiário. Procedimentos de baixa qualidade podem apresentar insucesso em um curto período de tempo, trazendo insatisfação para o paciente, desgastando a imagem do profissional e da OPS ${ }^{11}$. A auditoria é uma importante ferramenta de gestão da qualidade dos procedimentos executados.

Uma pesquisa em uma cooperativa odontológica verificou que os restauradores $(50,1 \%)$ foram os procedimentos que apresentaram maior quantidade de glosas, seguidos das raspagens $(19,4 \%)^{6}$. Outro trabalho do mesmo autor ${ }^{12}$ inferiu que não houve glosas indevidas, pois os critérios para a realização da glosa baseavam-se nas normas atuais da prática odontológica e na cobertura contratada pelos pacientes.

Um levantamento realizado em uma operadora odontológica avaliou 150 relatórios decorrentes de avaliações clínicas, tendo $29,33 \%$ sido considerados não conformes. Foram analisados 365 procedimentos; destes, os restauradores representavam $81,36 \%$; os selantes, $6,02 \%$; e os $12,62 \%$ restantes foram divididos entre raspagem, endodontia, radiografias, profilaxia, aplicação de flúor, exodontia, etc $^{4}$. Esse levantamento não apresentou semelhança com os dados encontrados no presente trabalho.

A prótese e endodontia representaram, respectivamente, $0,78 \%$ e $1,78 \%$ do total de procedimentos faturados. Do total de glosas, a endodontia e a prótese tiveram $4,77 \%$ e $2,32 \%$, respectivamente, diferente, portanto, do achado de uma pesquisa ${ }^{6}$ que encontrou $2,2 \%$ para a primeira e $1,8 \%$ para a segunda.

Apesar da baixa realização desses procedimentos, essas especialidades obtiveram a maior frequência de glosas, analisando-se cada grupo do rol individualmente. Com base na análise da Tabela 3 e da Figura 1, é possível observar que $24,37 \%$ dos procedimentos da prótese e $22,23 \%$ da endodontia foram glosados, não existindo diferença significativa entre ambas, mas sendo estas significativamente maiores que as demais. Esse resultado pode estar relacionado com a maior complexidade técnica dessas especialidades.

As falhas técnicas dos procedimentos protéticos estavam relacionadas, principalmente, com a confecção do núcleo metálico fundido, a falta de contato proximal das restaurações, a descontinuidade do dente/restauração e a desadaptação. Observou-se, também, a solicitação de tratamento protético em dentes sem condições clínicas para a permanência na cavidade bucal.

As glosas técnicas dos procedimentos endodônticos ocorreram devido a falhas relacionadas à indicação do tratamento, como: dentes com ruptura de assoalho da câmara pulpar, extensa lesão periodontal e resto radicular sem estrutura remanescente para receber tratamento restaurador. Também foram observadas intercorrências relacionadas à execução do procedimento: perfuração, fratura de lima, inadequação da ampliação do canal, da condensação lateral e do nível da obturação, como mostrou recente pesquisa ${ }^{13}$.

Atualmente, as OPS têm políticas diferentes, com requisitos e critérios, dependendo da empre$\mathrm{sa}$, os mais variados ${ }^{14}$. Isso porque as diretrizes clínicas odontológicas são realizadas pelas próprias 
OPS, que, apesar de apresentarem semelhanças, não possuem unicidade.

A auditoria necessita de critérios, que são declarações explícitas que definam o que está sendo medido, ou seja, um resultado mensurável de atendimento. Precisa, também, de um padrão, que é o limiar do cumprimento esperado para cada critério. Assim, surgem recomendações de diretrizes de prática clínica que podem ser usadas para desenvolver critérios e padrões ${ }^{15}$.

A criação de um protocolo único com diretrizes clínicas odontológicas realizado em parceria com as entidades de classe, associações da categoria, OPS e ANS poderia fornecer um padrão de qualidade para a prática odontológica para evitar as glosas e os conflitos entre a rede credenciada e a OPS. A ANS e a Associação Médica Brasileira apresentaram, em 2012, as diretrizes clínicas para medicina suplementar, alinhadas às práticas e aos princípios da medicina baseada em evidência. $\mathrm{O}$ objetivo de estabelecer diretrizes a serem seguidas no setor suplementar é viabilizar a qualidade da assistência.

A escassez de publicações sobre auditoria odontológica e as diferenças de metodologia e amostras das pesquisas encontradas dificultam a análise comparativa. Por isso, novas pesquisas na área são importantes. Além disso, é interessante que outras operadoras também divulguem seus dados para que a classe odontológica fique ciente dos principais motivos de glosa, evitando tais falhas.

Uma das limitações do presente trabalho foi a falta do padrão ouro nas avaliações técnicas. Entretanto, a equipe de auditores foi devidamente orientada e calibrada pela diretriz clínica interna da operadora, o que possibilitou a uniformização na análise dos dados.

Quando utilizada de forma eficiente, a auditoria resulta em grandes benefícios para pacientes e profissionais, garantindo o melhor uso da fonte de recursos, avaliando e melhorando continuamente a qualidade dos sistemas de saúde ${ }^{16,17}$.

\section{Conclusão}

A frequência total de glosa na OPS foi de 8,20\%. Os procedimentos mais glosados foram de radiologia $(35,93 \%)$, dentística $(24,04 \%)$ e prevenção $(16,70 \%)$. A quantidade de procedimentos glosados foi diretamente proporcional aos procedimentos executados. Quando se avaliou a frequência de glosa dentro de um mesmo grupo, a prótese $(24,37 \%)$ e a endodontia $(22,23 \%)$ foram as especialidades mais glosadas. Pesquisas na área de auditoria e a criação de diretrizes clínicas odontológicas para o setor de saúde suplementar são necessárias para esclarecer os critérios de glosa, diminuir as falhas técnicas e orientar os dentistas e as OPS sobre como obterem melhor qualidade na prestação de serviços.

\section{Abstract}

Objective: to observe the frequency of full disallowance and dental procedures performed by an accredited network of a Health Plan Provider. Materials and method: cross-sectional observational study in which we performed a retrospective survey in a group provider for dental care only, in the $3^{\text {rd }}$ quarter of 2012. A quantitative analysis pooled the procedures based on the List of the National Supplementary Health Agency. The collected data were tabulated and subjected to statistical analysis with 95\% confidence intervals, and significance level of 5\%. Results: from the 264,758 procedures billed, 21,716 were disallowed, so the overall percentage of disallowances over the total number of procedures was $8.20 \%$. The higher frequency of disallowances was seen in radiology (35.93\%), followed by dentistry $(24.04 \%)$, and prevention (16.70\%). Endodontics and prosthesis were $4.77 \%$ and $2.32 \%$ respectively, from the total of disallowances. However, when we individually analyzed each group from the List, $24.37 \%$ of the prosthesis procedures, and $22.23 \%$ of the endodontic procedures were disallowed. Conclusion: the overall frequency of disallowance in the Health Plan Provider was $8.20 \%$. The most disallowed procedures were radiology, dentistry, and prevention. When the frequency of disallowance within the same group was assessed, prosthesis and endodontics were the most disallowed specialties.

Keywords: Dental audit. Supplementary health. Health services evaluation. Forensic Dentistry.

\section{Referências}

1. Brasil. Agência Nacional de Saúde Suplementar [online]. Rio de Janeiro: ANS; 2013 [citado 2013 Jul 24]. Disponível em URL: http://www.ans.gov.br/aans/quem-somos/historico.

2. Brasil. Agência Nacional de Saúde Suplementar. Planos odontológicos: evolução, desafios e perspectivas para a regulação da saúde suplementar. Rio de Janeiro: ANS; 2009.

3. Brasil. Agência Nacional de Saúde Suplementar. Duas faces da mesma moeda: microrregulação e modelos assistenciais na saúde suplementar. Rio de Janeiro: ANS; 2005.

4. Juhás R. Auditoria em Odontologia. 2. ed. São Paulo: Viart; 2006.

5. Zimmermann RD, Paula FJ, Silva M. Vistorias em convênios e credenciamentos. In: Silva M, Zimmermann RD, Paula FJ. Deontologia odontológica: ética e legislação. São Paulo: Santos; 2011. p. 209-19.

6. Bragança DPP, Daruge Júnior E, Queluz DP, Fernandes MM, Paranhos LR. Avaliação dos procedimentos clínicos mais glosados nos convênios odontológicos. Rev Fac Odontol Univ Passo Fundo 2011; 16(2):136-9.

7. Moimaz SAS, Ayach C, Garbin CAS, Saliba O. Auditoria na saúde: justificativas de glosas no setor odontológico. J Health Sci Inst 2012; 30(2):112-6.

8. Colussi CF, Nickel DA, Calvo MCM, Caetano JC, Silva ACB. Análise da evolução da produção de procedimentos odontológicos de média e alta complexidade na rede de serviços públicos em Santa Catarina. Cad Saúde Colet 2009; 17(4):939-50. 
9. Hellen-Halme K, Johansson PM, Hakansson J, Petersson A. Image quality of digital and film radiographs in applications sent to the Dental Insurance Office in Sweden for treatment approval. Swed Dent J 2004; 28(2):77-84.

10. Naegele ER, Cunha-Cruz J, Nadanovsky P. Disparity between dental needs and dental treatment provided. J Dent Res 2010; 89(9):975-9.

11. Costa MT, Alevato H. Auditoria odontológica: uma ferramenta de gestão em saúde suplementar. In: VI Congresso Nacional de Excelência em Gestão [online]; 2010 Ago 5-7; Niterói. Anais eletrônicos. Niterói: Excelência em Gestão; 2010 [citado 2013 Ago 9]. Disponível em URL: http://www. excelenciaemgestao.org/Portals/2/documents/cneg6/anais/ T10_0315_1184.pdf.

12. Bragança DPP, Queluz DP, Fernandes MM, Lima SHR, Francisquini Júnior L, Daruge Júnior E. Verificação da viabilidade da auditoria presencial em operadoras de planos. Odonto 2011; 19(38):53-60.

13. Miranda GE, Kobata CM, Siqueira MC, Godói RMB, Daruge Júnior E, Francisquini Júnior L. Auditoria odontológica: intercorrências endodônticas e o esclarecimento ao paciente. Rev Fac Odontol Univ Passo Fundo 2012; 17(3):314-8.

14. Levin R. Dealing with dental insurance. Dent Assist 2009; $78(2): 20-1$

15. Lokuarachchi SK. Clinical audit: what is it and how to do it? Galle Med J 2006; 11(1):41-3.

16. Shankar AN, Shankar VN, Praveen V. The basics in research methodology: the clinical audit. J Clin Diagn Res 2011; 5(3):679-82.

17. Malleshi SN, Joshi M, Nair SK, Ashraf I. Clinical audit in dentistry: from a concept to an initiation. Dent Res J 2012; 9(6):665-70.

Endereço para correspondência:

Geraldo Elias Miranda

Rua Conde Ribeiro do Vale, 105/303 -

Sagrada Família 31.030-470

Belo Horizonte/MG, Brasil

Fone: (31) 3785-1369 (31) 9871-1369

E-mail: geraldoelias@hotmail.com

Recebido: 14/08/2013. Aceito: 07/10/2013. 